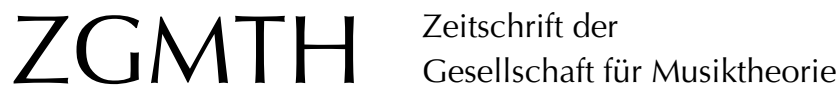

Klassen, Janina (2003/05): Musik, Körper und Zeichentheorie - Notizen zu Naomi Cumming, The Sonic Self. Musical Subjectivity and Signification, Bloomington and Indianapolis: Indiana University Press 2000. ZGMTH 1-2/2/2-3, 288-303.

https://doi.org/10.31751/209

(C) 2003/05 Janina Klassen

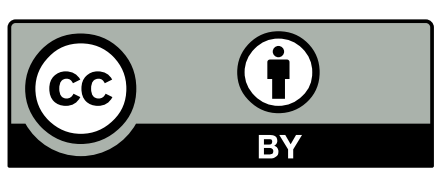

Dieser Text erscheint im Open Access und ist lizenziert unter einer Creative Commons Namensnennung 4.0 International Lizenz.

This is an open access article licensed under a

Creative Commons Attribution 4.0 International License.

veröffentlicht / first published: 01/04/2005

zuletzt geändert / last updated: 01/12/2008 


\section{Musik, Körper und Zeichentheorie. Notizen zu Naomi Cumming, The Sonic Self. Musical Subjectivity and Signification, Bloomington and Indianapolis: Indiana University Press 2000}

Wie gewinnt eine Violinschülerin `Persönlichkeits? Wie soll `Emotionalität im Spannungsfeld von Autorenintention, Werkautonomie und persönlichem Erleben musikalisch erarbeitet werden? Welchen Anteil hat der eigene Körper am Gelingen der Aufführung? Was nützt das angesammelte Wissen? Wo klingen die bewunderten Vorbilder durch? Worin unterscheiden sich Virtuosität und Sport? Mit diesen unlösbaren Fragen aus ihrer eigenen Studienzeit führt Naomi Cumming gleichsam anekdotisch ein in Grundprobleme von Musik, Interpretation und Performance. Was eine ımusikalische Persönlichkeit nun tatsächlich ausmacht, bliebe bei allen, die ihr Fehlen wortreich einklagten, vage, mystisch und »frustratingly undefined" (S. 31). An die Entdeckung unbekannter eigener Ausdruckspotentiale (»voices«) beim Spiel knüpft Cumming Überlegungen zur Identität des durch Töne artikulierten smusikalischen Ich،. Sie geht davon aus, daß sensorische Selbstwahrnehmung und Feedbackerfahrungen bei der künstlerischen Selbstkonstruktion zusammenwirken. Die ıIdentität der musikalischen Persönlichkeit setzt sich aus äußeren und inneren Komponenten zusammen. Als säußere r nennt Cumming den individuellen Ausführungsstil, den spezifischen Sound und die persönliche Gestik, die allerdings sämtlich nicht unabhängig von musikalischen Konventionen existieren, während die sinneren der Introspektion angehören, der Welt der Gedanken, Träume und Fantasien. Bei der Introspektion fällt die Entscheidung, warum ein bestimmtes Timbre gewählt wird (S. 59). Wie Charles S. Peirce und Michael Bachtin geht Cumming von einem dialogischen musikalischen Ich aus. Zur motorischen, sensorischen und emotionalen Erfahrung gehört außerdem das analytische und historische Wissen über Musik. Ohne explizit den mediengeschichtlichen Anteil zu betonen, bezieht Cumming in ihren Fallbeispielen auch die auf Schallplatten und CD gespeicherten Musikeinspielungen ein. Eine zentrale Rolle spielt dabei die Sprache, in die Musik transformiert, durch die sie analysiert, in der sie reflektiert und kommuniziert wird. Bekanntlich klaffen zwischen musikalischem Erleben und theoretischen Befunden oft $\mathrm{Ab}$ gründe. Sprache formt das Musikverständnis. Deswegen gehören Sprachanalyse und Sprachkritik zum festen Bestand der Theorie.

Man kann das Buch lesen wie einen ausführlichen, umsichtigen, faktenreichen und sehr inspirierenden Essay. Spannend geschrieben und variantenreich formuliert bietet es einen höchst informativen Parcours durch die derzeitigen Themen nicht nur der $>$ New Musicology und der angrenzenden Gebiete von Musik- und Wahrnehmungstheorie, Emotionsund Erlebensforschung, sondern auch der Aufführungspraxis und Interpretation. Der im Buch zusammengedrängte Reichtum an Themen und Perspektiven hätte Stoff für gleich mehrere Bände geboten, da jedes der angesprochenen Gebiete für sich eine umfassende Diskussion erfordert. Im Vergleich zur deutschen Wissenschaftsprosa ist das didaktische Konzept allerdings ungewohnt. Die Autorin präsentiert ihre Gedanken sozusagen in drei Schwierigkeitsgraden und setzt dabei unterschiedliche Akzente. David Lidovs Hinweis, daß die ersten zwei Drittel auch von NichtMusiktheorie-Experten verstanden werden könnten (Vorwort S. XV), gebe ich gern mit Hochachtung vor Lidovs imaginierten Lesern wieder. Daß die Leser aufgrund des pragmatischen Ansatzes sofort mit einbezogen werden, erhöht zwar die Neugier und das eige- 
ne Engagement. Trotzdem empfinde ich die Lektüre aufgrund der Vielfalt der Ansätze sowie der gedanklichen Intensität der Darstellung von Beginn an auch als besondere Herausforderung. Überspringen lassen sich selbst für gut informierte Leser die einzelnen Kapitel nicht, da sie aufeinander aufbauen. Daß die Themen weitgehend an artifizieller (Violin-) Musik des 18. und 19. Jahrhunderts expliziert werden, entspricht immer noch unserer heutigen Konzertpraxis und dem Kernrepertoire an Musikhochschulen. Hier geht die Autorin von ihrer eigenen Erfahrung aus und sie greift auch immer wieder konkret darauf zurück. Diese Nähe erhöht die Anschaulichkeit ihrer Darstellung entschieden. Sie fordert allerdings auch immer wieder eine Auseinandersetzung mit älteren Konzepten von Werktreue und Hermeneutik heraus. Trotzdem läßt sich auch wegen der grundsätzlichen Überlegungen zu Wahrnehmung und Performance viel daraus gewinnen für die Auseinandersetzung mit Neuer Musik.

Bei der Diskussion des Komplexes 'Ton<, der eine eigene Qualität als Zeichen erhalten soll, um ihn in der Interpretations-Analyse von der Körperlichkeit der Performer, vom Instrument, vom Raum und von der individuellen Erwartung abtrennen zu können (Kap. 4, S. 123 ff., hier erörtert am Violinton), ist es hilfreich, dem knappen Hinweis auf die Musique concrète ausführlicher nachzugehen. Die Konstruktion eines skörperlosen Klangs, der zunächst durch elektroakustische Bearbeitung natürlich produzierter Töne und später durch rein elektronisch generiertes Material entsteht, läßt sich an frühen Stücken von Pierre Schaeffer oder Pierre Henry sehr sinnlich und ganz unmittelbar nachvollziehen. Umgekehrt bietet Helmut Lachenmann mit seinem spezielleren Konzept einer Musique concrète instrumentale anschauliche musikalische Beispiele gerade für die Poesie der `Ränderı, der Nebenund Begleitgeräusche der Tonerzeugung, die ebenfalls eine eigene Zeichenhaftigkeit erhalten können. Davon unabhängig hat Roland Barthes diesen Aspekt in seinem vielzitierten Essay über die »Rauhheit der Stimme« $\left({ }^{3} 1991\right)$ am Beispiel von Schumann-Interpretationen auf der Basis eines zeichentheoretischen Ansatzes literarisch thematisiert und mit Julia Kristevas Idee eines `Phäno-` und `GenoGesangs verbunden. Diese Musik- und Lektüreerfahrungen erleichtern die Abstraktion vom gewohnten ästhetisch-programmatisch geformten sschönen Ton nach der Konvention des 18. und 19. Jahrhunderts. Sie schärfen das Bewußtsein für Cummings differenzierten Interpretationsansatz.

So bin ich während der Lektüre immer wieder ausgeschert, einmal, um mich genauer zu informieren, etwa über die Zeichentheorie von Peirce, dann auch, um einzelnen hier angestoßenen Gedankengängen weiter nachzugehen, die Referenzquellen sowie zusätzlich neuere Forschungsliteratur heranzuziehen und die im Buch verschriftlichten Ansätze mit hiesigen Fachdiskussionen zu konfrontieren. Auf diese Weise erhielt die Auseinandersetzung mit Cummings Ideen in den mehrmonatigen Lesegängen immer wieder auch andere Schwerpunkte, deren Erarbeitung sich nach und nach verselbständigte. So entstand eine Fülle weiterer Wissensinseln um den reichhaltigen Grundtext von Cumming herum, bis eine freundliche Stimme mich zum Loslassen mahnte. Am Ende gingen die vielen wertvollen Fragmente, Notizen und Anmerkungen auf der Strecke zwischen Berlin und Freiburg verloren. Das Buch dann noch einmal zu lesen, nun vor dem Hintergrund eigener neuer Lektüren und Unterrichtserfahrungen ${ }^{1}$, öffnete wiederum andere Perspektiven, insbesondere auf die Sprach- und Zeichentheorie sowie auf die , Ästhetik des Performativen $\iota^{2}$. Im folgenden gebe ich daher zuerst einen Überblick über Cummings Buch, um dann allgemeiner auf die Punkte 'Körper und `Zeichen s einzugehen.

\section{Übersicht}

In der Einleitung eröffnet Cumming ein ganzes Spektrum fundamentaler Diskurse, die in ihrer Tragweite über die engeren Grenzen von Musikforschung hinausragen. Fragen nach 
dem Verhältnis abstrakter Systeme und konkreter Bezüge, hier thematisiert am Beispiel von metasprachlichen Analysesystemen, narrativer Beschreibung, metaphorischer Bedeutung und individuellen Verstehensweisen, gehören ebenso zu allgemeinen epistemischen Grundlagen wie die philosophische Auseinandersetzung mit Subjektivität und Objektivität, Emotionalität und Selbstkonstruktion, die unerschöpfliche body-and-mind-Diskussion, Interpretationsfragen, neurobiologische Ansätze oder - allgemeiner - das Zusammenwirken von Psychologie, Kognitions-, historischer und systematischer Musikwissenschaft. Vieles davon beherrscht seit langer Zeit schon die wissenschaftlichen Diskurse, manches scheint längst sabgehaktı zu sein, wie die Übertragung der Zeichentheorie auf Musik, anderes, wie die Körper-Debatte, gilt vielen hauptsächlich als modisch. Cummings Buch fesselt indessen gerade deshalb so sehr, weil sie keine kaleidoskopartige Rundschau der `New Musicology anbietet, sondern demonstriert, wie konstruktiv eine erneute Auseinandersetzung mit diesen Fragen für die aktuelle Situation sein kann. Daß bestimmte Themen innerhalb der Wissenschaftsgeschichte immer wieder aufgegriffen werden, liegt einmal in der Sache begründet. Ein Vorgang wie die abstrahierende Formalisierung der Zeichensysteme fordert jedes Mal neu dazu heraus, das Verhältnis des Analyseinstruments zum konkreten Objekt zu definieren. Andere Themen, wie die body-and-mind-Diskussion, drängen dann besonders in den Vordergrund, wenn die an Musik gerichteten Erwartungen, Bedürfnisse und Erkenntnisinteressen sich deutlich zugunsten einer Seite verschieben, wie in den letzten Jahrzehnten in bezug auf die Rolle von Körper und Emotion. Mit der body-and-mindDiskussion und der Zeichentheorie fixiert die Autorin gleich zu Beginn als zwei wichtige Leitfäden, die sich durch das ganze Buch ziehen. Der Antagonismus von Körper und Geist wird deshalb verworfen, weil er eine Abwertung sensorischer Komponenten impliziere. Cumming greift damit eine bereits im 19. Jahrhundert begonnene Debatte auf, an der un- ter anderem Peirce kritisch teilnahm. Peirces Zeichentheorie, die schon einmal (vor mehr als zwanzig Jahren) eine Renaissance erfahren hat, wird von Cumming nun unter anderer Perspektive wieder eingeführt. Einmal schließt sie sich der traditionellen Absicht an, durch die zeichenhafte Formalisierung musikalischer Vorgänge eine gleichsam ıneutralere، Position zu ermöglichen, als die üblichen metaphorischen Beschreibungen oder die eingefahrenen Analyseschemata bieten, um sowohl ein vorzeitiges Aufladen mit »interpretable meaning" (S. 18) zu unterbinden als auch gestische oder haptische Vorgänge gleichrangig zu Notation und Klangqualität in die Reflexion einbeziehen zu können. Diese letzten Punkte gehören allerdings zu den erst noch zu realisierenden Visionen Cummings. Zum anderen gewinnt sie der Dynamik von Peirces prozeßorientierter Theorie neue Zugangsweisen ab. Als übergeordnetes Ziel des Buches soll gelten, den Abstand zwischen «academic philosophy and academic music theory« zu überbrücken, so David Lidov im Vorwort (S. XV). In deutsche Verhältnisse übersetzt müßten vielleicht die Abstände zwischen historischer und systematischer Musikwissenschaft, philosophischer Ästhetik, Musiktheorie, -psychologie und Musikerziehung genannt werden.

Nach der Einführung in die oben skizzierten Diskurse erhält man im ersten Kapitel einen Einblick in die Themenkreise Körper und Zeichen vor allem aus der Perspektive der produzierenden Künstler, im zweiten wechselt der Standpunkt auf die Rezipientenebene. Das dritte Kapitel bietet dann einen ersten systematischen Einstieg in die Zeichentheorie von Peirce, die im vierten Kapitel, in dem die Körper-Diskussion aus dem ersten wieder aufgegriffen wird, um Wahrnehmungsvorgänge bereichert ist. Daran schließt sich eine intensivierte Diskussion über Gestik, Kinästhetik und Interpretation an (Kap. 5). Das 6. Kapitel dreht sich um Grundsatzfragen analytischer Systeme vor dem Hintergrund subjektiver Erfahrung. Im 7. bis 9. Kapitel (»Complex Synthesis«) werden die vorher ausgebreiteten Bereiche dann auf diesem Fundament verknüpft, 
gleichzeitig noch einmal differenziert und nun vor dem Hintergrund der New Musicology neu diskutiert.

Eine Handlungsanweisung, wie nun künftig mit Musik theoretisch umzugehen wäre, erhält man allerdings nicht. Die Autorin plädiert zwar nachdrücklich für sorgfältige musik- und zeichentheoretische Analysen, die, jede für sich, zuerst vorgenommen werden müßten (S. 225), sie führt selber aber keine entsprechende >Musteranalyse durch, in der sie die Beziehung zwischen musikalischer Struktur, Körperspuren und ästhetischem Verstehen systematisch zerlegte. Vielmehr geht Cumming in ihren Exempeln mit Absicht auf die Ebene einer gleichsam svortheoretischen Literarisierung zurück, um die Illusion eines wissenschaftlich eindeutigen Verstehens von Musik auszuschalten. Sie praktiziert damit die Synthese ihrer eigenen im Buch explizierten Problemfelder (s. S. 225-32). Indessen wächst gerade durch die erzählende Prosa die Verantwortung für die narrativen, metaphorisch sensibilisierten wortsprachlichen Erläuterungen erheblich. Genau diese sprachliche Sensibilisierung gilt als ein wünschenswertes Ziel (vgl. S. 304). Nur so kann nach Cumming der pragmatische Ansatz gelingen, mit dem aus den Analysen gewonnenen theoretischen Wissen um die Komplexität musikalischer Vorgänge, Strukturen oder Konstruktionen immer wieder auf das konkrete Ereignis, die erklingende Musik mitsamt ihrer Fülle an affektiven Assoziationen, zurückzugehen. Damit entscheidet sie sich in der bis ins 19 . Jahrhundert zurückreichenden Grundsatzfrage zum Verhältnis von erzählter Geschichte und exakter Wissenschaft eindeutig für die Tradition literarischer Erzählung. In diesem Sinne verteidigt sie auch eine subjektive, als rein private Äußerung deklarierte Lesart, wie sie Hans Heinrich Eggebrecht für sich beansprucht (S. 255 ff). Dabei scheint mir allerdings zu kurz zu kommen, daß eine gedruckte Meinung bekannter Experten - und sei sie noch so ssubjektiv - gleichwohl übergreifende Autorität ausstrahlt und nicht länger privat ist.
An Hermann Kretzschmar erinnert Cummings Verfahren, Musik metaphorisch zu personalisieren, um die komplexe musikalische Emotion und Expressivität sprachlich benennen zu können (s. zur Konstruktion einer »persona« als »agency« für Expression S. 205ff. und S. 249ff.). Die metaphorische Personifizierung von Musik, die klingend mitteilt, erfolgt in der Absicht, das flüchtige, immaterielle und dabei höchst wirkungsvolle Phänomen Musik greifbar zu machen. ${ }^{3}$ Anders als Kretzschmar, der aus ähnlichem Anlaß vor hundert Jahren an den Grundlagen einer beschreibenden musikalischen Hermeneutik meißelte, kann Cumming auf eine breite Forschungspalette zurückgreifen. Sie wählt ein offenes Konzept. Dabei macht sie sich einen aus der sogenannten `Fuzzy<-Logik ${ }^{4}$ bekannten Effekt zunutze, nämlich die Einsicht, daß eine unscharfe Logik realen Erscheinungen beziehungsweise menschlichen Verhaltensweisen näher kommt als exakte Schlüsse. Für die Rezipienten entfällt damit die einschüchternde Zumutung, Musik im analytischen, das heißt hier auf der Idee autonomer Eigengesetzlichkeit basierenden, Sinne srichtig « zu verstehen. ${ }^{5}$ Gleichwohl funktioniert diese Logik in der Anthropologie auch nur aufgrund präziser Methoden. Ohne seriöse theoretische Vorarbeiten setzt sich Musikbeschreibung dem Vorwurf postmoderner Beliebigkeit aus. Dementsprechend fordert (und bietet) auch Cumming punktuell Einblicke in ihre detaillierten musik- und zeichentheoretischen Analysen als Basis für ihr narratives Vorgehen, das sie dabei selbstkritisch reflektiert. Womöglich wirkt ihr Buch gerade deshalb so inspirierend.

\section{Positionen}

Die hier gestellten Fragen gehören zu den grundsätzlichen aller mit Musik befaßten Wissenschaften. Unabhängig von ihrem historischen Alter wird Musik immer wieder neu gehört, und sie gewinnt, wenn sie aktuell erklingt, hier und jetzt für Ausführende wie Rezipienten individuelle, oft emotionale Bedeu- 
tung, deren Spannbreite von emphatischem Miterleben und angenehmer Stimmung über Langeweile und Frustration bis hin zu vehementer Ablehnung reichen kann. Daß sich diese Bedeutung mit der Zeit und abhängig von der jeweiligen Situation wandelt, gehört zu Erfahrungen, die bereits an der eigenen Biographie nachvollzogen werden können. Umso anspruchsvoller gerät der Versuch, verschiedene historische Kontextualisierungen zu rekonstruieren. Cumming nimmt als Ausgangsbeispiel, an dem sie die Körpererfahrung der Spielerin diskutiert, die Violinsonate g-moll op. $1 \mathrm{Nr} .10$ von Giuseppe Tartini. Im 19. Jahrhundert erhielt das Stück den programmatischen Beinamen >Didone abbandonatas, der auf das Bedürfnis einer inhaltlichen Orientierung verweist. Heute hören wir die Sonate als autonome Musik. Indessen entstammt das 1732 publizierte Stück historisch wiederum einem anderen, nämlich anwendungsbezogenen Kontext. Danach soll Musik eine emotionale Wirkung erzielen. Der Übertragungsvorgang funktioniert nach den Vorstellungen der Zeit mechanisch, indem die Schwingungen der Saiten beziehungsweise Luftsäulen die Fibern des Herzens in analoge Bewegung versetzen. Doch gelingt es trotzdem nicht, mit der Aufzählung einzelner Affekte oder einem Partitur-Mapping durch die Applikation diverser musikalisch-rhetorischer Figuren das zeitgenössische Erlebnis zu rekonstruieren, da das in der damaligen Theorie festgehaltene Wissen nur sehr unzureichend Rückschlüsse auf das Erleben von Musik wiedergeben kann ${ }^{6}$, selbst wenn man auf die von Cumming angesteuerte narrative Ebene geht. Nachdem inzwischen selbst in der historischen Aufführungspraxis die Suche nach einer authentischen Interpretation und der Wunsch nach einem korrekten Verständnis des geschichtlich gewordenen swahren Sinns` (Gadamer) ${ }^{7}$ fallengelassen worden sind, dürfte dieser Aspekt heute auch in den ästhetischen Debatten keine wesentliche Rolle mehr spielen. Weder die jetzige noch die zeitgenössische Emotionalität des Stücks (die beide nicht identisch sind) können mit der
Sprache musikalischer Analyse tatsächlich erfaßt werden.

Analysen erschließen Ordnungsstrukturen und Konstruktionsprinzipien. Dabei setzen sie die Idee des autonomen Werkes vielfach stillschweigend voraus. Sie zielen auf allgemeingültige, werkästhetische Aussagen und fußen auf der Auslegung des von den Komponierenden verschriftlichten Sinns ${ }^{8}$ in der Partitur (zumindest im gewohnten Repertoire des 17. bis 19. Jahrhunderts). Sie blicken sozusagen aus der Innenperspektive, während das emotionale Erleben der Hörer, die Außensicht, kaum mit Fragen an die Partitur zu erforschen ist, wenn die intendierte Wirkung nicht gleichgesetzt werden soll mit der tatsächlichen Reaktion. Zwar enthalten die unterschiedlichen, rezeptionsgeschichtlich erschließbaren Aufführungsstationen beziehungsweise -situationen der Tartini-Sonate auch abstrahierbare Erlebniskonstituenten. Die liegen begründet in der kulturellen Tradition etwa bestimmter charakteristischer Rhythmen, des temperierten Tonsystems und der dafür charakteristischen Obertonspektren, der Konventionalität musikalischer Formeln ('Seufzer`-Motive) oder der Gewöhnung an einen bestimmten, kultivierten Geigenton. Trotzdem werden in beiden Fällen, dem wirkungs- wie dem werkästhetischen Ansatz, unterschiedliche Sprachrepertoires verwendet, die auf jeweils verschiedenen Ausgangspositionen und Zielen beruhen. Hier stehen der metaphernreichen Erlebnisbeschreibung und den vom Einzelfall ausgehenden Untersuchungen die abstrakteren (gleichwohl metaphorischen), auf generelle Strukturen abhebenden analytischen Metasprachen tatsächlich oft schwer vereinbar gegenüber. Cumming bezieht sich dabei auf den psychologischen Ansatz von Meyer und Narmour auf der einen sowie auf die Theorien von Lerdahl/Jackendoff oder Raffman (und deren kognitionswissenschaftlichen Ansatz) auf der anderen Seite. ${ }^{9}$ Einen spannenden Sonderfall bilden in diesem Zusammenhang Heinrich Schenkers ,Urlinien «. Als graphische Zeichensysteme reduzieren sie die Stücke auf wenige zusammenfassende Aspekte. Im Vor- 
dergrund steht jedoch nicht die übliche Absicht, zu kontextenthobenen Anschauungen zu gelangen, wofür Diagramme im allgemeinen methodisch eingesetzt werden, sondern die Graphik soll gerade das Individuelle der jeweiligen Komposition übersichtlich demonstrieren (vgl. Cummings Kommentar S. 171ff.).

Methodisch ist die Differenzierung zwischen musikalischem Erlebnis und Analyse angebracht, da sie sich auf zwei sehr unterschiedliche Komponenten beziehen. Probleme und wechselseitige Frustrationen treten auf, wenn erwartet wird, daß die Vielfalt aller unter $>M u-$ sikı versammelten Phänomene erstens mit einer Art Superformel zu erfassen und zweitens durch einen singulären Text darstellbar wäre. Zur Vertiefung der Gräben dürfte außerdem der (oft nur implizit, aufgrund der Deutungshoheit, erhobene) Absolutheitsanspruch jeder Seite beigetragen haben. Nun zeigt die Evaluierung der Analyseinstrumente und -sprachen sowohl bei der musikalischen Emotionsforschung als auch bei den werkanalytischen Fragen, daß sich aus der Wissenschaftstheorie vertraute binäre Oppositionen wie die Denkfiguren body/mind, Subjekt/Objekt, Emotion/ Struktur, Aufführung/Text, konkret/abstrakt in den letzten Jahren erheblich verschoben, wenn nicht sogar aufgelöst haben. Inzwischen läßt sich im historischen Rückblick auch eine deutliche Änderung des erkenntnistheoretischen Fokus beobachten. Dafür gibt die Rezeption von Peirces Zeichentheorie ${ }^{10}$ ein anschauliches Beispiel, das auch für Cummings Vorgehen charakteristisch ist. Anstelle einer Anbindung an kognitionswissenschaftliche Kriterien, die auf das Herausarbeiten allgemeiner Gesetzmäßigkeiten zielt, gilt das Interesse dem konkreten Einzelfall mit seinen emotionalen Komponenten. Beide Optionen lassen sich zeichentheoretisch kodieren. Über das Erleben hinaus interessiert inzwischen generell der körperliche Anteil an Sprache beziehungsweise an Musik. Wie Lakoff/Johnson in ihrer Metapherntheorie zeigen, reicht er bis in die Sprachbilder hinein. Diese von Cumming verfolgte Interessenverschiebung ist Teil einer allgemein zu beobachtenden Ten- denz in den Kunstwissenschaften. »Je kälter der Blick wird, den die moderne Wissenschaft auf die Sprache wirft, desto größer wird die Sehnsucht nach Licht und Wärme«, so referiert Jürgen Trabant ${ }^{11}$ in Anlehnung an Humboldts energetisches Modell 1986 ein Bedürfnis, das bewußt an einen Teil der sanderen Moderne anknüpft und die Erforschung des lebendigen Gebrauchs von Sprache ins Zentrum rückt. Zur Medialität der Rede gehören neben dem Text eben auch Intonation, Gestik und Mimik. Dabei geht es nicht lediglich um die Verlagerung des Interesses von den Produktions- zu den Performanzstadien der Rede, die bereits die klassische Rhetorik prägt. Vielmehr steht längst grundsätzlicher das Verhältnis von Sprache und Sprechen zur Disposition $^{12}$ - eine Frage, mit der sich Peirce bereits Ende des 19. Jahrhunderts intensiv befaßt hat. Da erklingende Musik immer ästhetische Gegenwart ist, muß die Grundsatzfrage nach den aktuellen Hör- und Erfahrungsweisen immer wieder neu gestellt werden. Vor diesem Hintergrund erscheint auch die Neubewertung der Zeichentheorie in bezug auf Musik weniger als Symptom eines zu beobachtenden spost-linguistic-turn', sie öffnet vielmehr die Kulissen für eine Erweiterung der Perspektive. sörper steht hier auch nicht ausschließlich für das `Andere des Logos. Er gilt in diesen theoretischen Ansätzen sozusagen als das neue Mantra, das heißt, ,Körper soll all das einholen, was durch den autonomen Werkbegriff ausgeklammert wurde.

\section{Körper}

Musik ist ohne Körper nicht zu haben. Selbst Musik, die elektronisch generiert ist, wie Maryanne Amachers dense boogie 1 von 1998, erfordert Ohren, in denen die verschiedenen Frequenzüberlagerungen tatsächlich rechtslinks verschoben kitzeln, oder Körper, die Klang-Kompositionen im unterhalb der Hörschwelle liegenden Tieftonbereich erspüren. Die mediale Verstärkung von Klang ermöglicht Hörerfahrungen, bei denen Musik weit 
intensiver körperlich wahrgenommen werden kann (oder sogar zu werden droht) als bei herkömmlichen Aufführungen. Welche starken physischen Erfahrungen erwartet werden, zeigt die spontane Reaktion vieler Hörer, die Ohren zu schützen, sobald die Regler aufgehen. ${ }^{13}$ Mit der Möglichkeit, Musik aufzuzeichnen und beliebig wieder abzuspielen, ist die zeitliche und räumliche Abkopplung von Musikmachen und Hören möglich geworden. Der Gesang erreicht das Ohr der Zuhörer nicht mehr unmittelbar, sondern existiert körperlos als analog oder digital gespeicherte Information, die jederzeit wieder abrufbar ist. Gleichzeitig transportiert die medial verselbständigte und erweiterte Stimme durch die akustische Präsenz tonproduzierender Begleitgeräusche oder elektronische Manipulationen die Illusion von Intimität. Während Künstlerinnen und Künstler verschiedener Sparten die neuen Reproduktionsmedien gezielt einsetzen, um Wahrnehmungsvorgänge artifiziell zu manipulieren und menschliche, dingliche oder künstliche Klangerzeugung elektronisch zu mischen, wird uns der Abspaltungsvorgang im Alltag, beim Hören von Radio, CD oder MP3, kaum mehr bewußt. Es scheint aber, daß die inzwischen flächendeckende »Trennung der Ohren vom Körper « ${ }^{14}$ heute vor allem die verstärkte Sehnsucht nach ganzheitlichem sinnlichem Erleben und das Bewußtsein für die Körperlichkeit von Musik auslöst.

Daß Körpereinsatz bei der Produktion von Musik unerläßlich ist, beschäftigt schon die frühchristliche Theologie intensiv. Auch die Reflexion über die Art und Weise dieses Einsatzes hat in unserer Geschichte eine lange Tradition. Neu ist vor dem Hintergrund der traditionellen Kunstdebatten in den hier diskutierten Themen dagegen die Umwertung der Aufführung. Sie wird zum eigentlichen Kunstereignis. Deshalb wird die Aufführung zum Gegenstand der Diskussion. Für alle Aspekte rund um die Aufführung gilt es daher, eine theoretische Grundlage zu schaffen, die eine Teilnahme an bereits bestehenden Diskursen ermöglicht. Wie im Theater, so läßt sich auch in Teilen der aktuellen Kunst eine herausfordernde `Rückgewinnung` des durch den Prozeß der Zivilisation immer gründlicher abgespaltenen Körpers (Norbert Elias) nachweisen. Inzwischen kann die von Carl Dahlhaus 1977 nachdrücklich vertretene These der "ästhetischen Gegenwärtigkeit»"15 von Musik (egal welchen Alters) dahingehend radikalisiert werden, daß Musik überhaupt erst durch körperliche Präsenz entsteht. Im Akt der Aufführung läßt sich die physische Präsenz der Ausführenden kaum ablösen vom Eigenwert des produzierten Klangs, als sinnliche Realisierung des in der Partitur kodierten musikalischen Werks. Im Gegenteil: gerade die untrennbare Mischung macht die immer wieder bestätigte höhere Attraktivität einer 'Live`Perfomance selbst technisch gelungeneren Studioproduktionen gegenüber aus. Folgt man Fischer-Lichte, so treten Künstler dabei nicht bloß als mediale Vermittler in Erscheinung, sondern als sembodied mind. Gemeint ist damit das einheitliche Zusammenwirken von körperlichen und geistigen Komponenten im Moment der Aufführung. Zuhörer und Zuschauer, die aktiv daran teilnehmen, erfahren an sich selber eben diese Einheit als ,Glücksmoment،, der ssüchtigs machen kann. ${ }^{16}$

Der alte Konflikt zwischen der Autorität der Partitur und der Dynamik des Performativen erfährt damit eine erfrischend neue Wendung, weil der Blick frei wird auf die in den ästhetischen Debatten weitgehend ausgeklammerten physischen Bedingungen von Kunst. Doch darf auch nicht unterschlagen werden, daß gerade die traditionelle Denkfigur einer binären Körper/Geist-Opposition, die sich in der Kunsttheorie ebenso rasch wie nachhaltig verselbständigt hat, in den Autonomiedebatten des 18. Jahrhunderts die Funktion erfüllt hat, eine Eigenständigkeit artifizieller Musik überhaupt erst behaupten zu können. Schließlich gehört das Autonomiekonzept deswegen zu den wichtigsten ästhetischen Errungenschaften, weil damit die Idee der Freiheit verknüpft worden ist. Musik symbolisiert aufgrund der Transparenz der Schallereignisse diese Freiheit. Kunst kann damit zur utopischen Gegenwelt werden. Körperli- 
cher Einsatz gilt auch im 18. Jahrhundert als Voraussetzung für Musikproduktion. Nur versucht man in zeitgenössischen Reflexionen wie etwa dem Versuch Carl Philipp Emanuel Bachs, die Aktion der Künstler ausschließlich auf die Übersetzung einer im Text verschriftlichten Autoren- beziehungsweise Werkintention zu fixieren und den nicht integrierbaren Überschuß an präsenter Körperlichkeit wegzurationalisieren. Bachs bekannte Beschreibung, daß der Pianist »nothwendig sich selbst in alle Affeckten setzen können [muß], welche er bey seinen Zuhörern erregen will $\ll^{17}$, beruht noch auf der Überzeugung eines analogen Schwingens von Tonfrequenzen und Nervenfasern. Andere Theorien entlehnen dafür den medizinischen Ausdruck der >Ansteckung ‘. ${ }^{18}$ Diesen mechanischen Vorgang müssen die Künstler auslösen. Damit das gelingt, sind mehrere Schritte notwendig. An erster Stelle stehen Studium und Analyse der Partitur, »um eine Einsicht in den wahren Inhalt und Affeckt eines Stückes zu erlangen« (Bach, S. 117), das heißt um das Stück und die darin enthaltene Absicht des oder der Komponierenden überhaupt erst kennenzulernen. In einem zweiten Schritt gilt es, sich seiner eigenen privaten Körperlichkeit völlig zu entledigen, um dann in einem dritten Schritt zu einem gleichsam semiotischen Körper zu werden und sich die Rolle einer musikalischen spersona des Stücks anzueignen. Die spersona sentspricht dem slyrischen Ich beziehungsweise den verschiedenen Autoren-Stimmen, die in einem Stück enthalten sein können. Den philosophischhistorischen Hintergrund für eine derartige Konstruktion des Künstlerkörpers bildet die auf Gottfried Wilhelm Leibniz zurückgehende :Zwei-Welten-Theorie ${ }^{19}$, nach der neben der tatsächlichen auch mögliche alternative Welten bestehen. Im konkreten Fall soll der Künstler in die aus dem Text gewonnene Bühnenfigur sozusagen physisch hineinschlüpfen. Gleichzeitig muß diese Form der Verkörperung ein rational kontrollierter Vorgang bleiben: eine vollkommene Verschmelzung der Identität von Künstler und Rolle ist nicht vorgesehen. Schließlich darf Othello Desdemona nicht wirklich töten ${ }^{20}$, so wenig wie Bachs imaginärer Klavierspieler tatsächlich in Trauer versinken darf, weil er sonst seine Aufgabe nicht erfüllen kann. Da Bachs Ausführungen auf Wirkung zielen, werden die emotionalen Gehalte von Mimik und Gebärden ausdrücklich in die Handlungsanweisungen mit einbezogen. "Man sieht und hört« dem Spieler an, was er erreichen will. ${ }^{21}$ Daher sollte er nicht starr »wie ein geschnitztes Bild vor dem Instrumente" hocken. "Gute», das heißt angemessene, Gebärden unterstützen die ästhetischen Absichten und kommen nicht nur »den Zuhörern zu Hülfe«, vielmehr helfen sie auch dem Spieler selber, seinen Vortrag zu optimieren und zugleich neue Ausdrucksmöglichkeiten zu entdecken. Viele erfahren dadurch mit Verwunderung, daß »ihre Werke mehr enthalten, als sie gewußt und geglaubt haben" (S. 123), berichtet Bach. Eine ähnliche Selbsterfahrung beschreibt noch Cumming in ihrem Ausgangsbeispiel, wo die Violinspielerin durch ihre Körperarbeit (Bogendruck, Armbewegung) hervorgerufene ungeahnte ,Stimmen (voices) in sich entdeckt.

In Werkkonzepten, in denen Musik nicht bloß als vermittelndes Medium von Effekten, sondern als autonome Kunst rezipiert werden soll, soll das Publikum zwar den semiotischen `Klangkörperı, nicht aber die reale Physis der Ausführenden wahrnehmen. Auf dem Theater helfen Maske, Kostüm und Bühnenbild, der fiktiven Figur mit ihren Affektzuständen und Verhaltensweisen zu folgen. Musikalische Aufführungen stehen hier vor weit größeren Problemen als dramatische, da die Einfühlung in das metaphorische Subjekt einer instrumentalen Fantasie oder Sonate eine weit radikalere Abstraktionsleistung fordert. Der mediale Vorteil von Musik, nämlich ohne den Umweg über Sprache aufgrund ihrer sensorischen Repräsentation unmittelbar Bedeutung zu gewinnen, ist gleichzeitig auch ihr größter Nachteil, wenn es darauf ankommt, eine ästhetische Idee zu übermitteln. Schließlich hört und sieht man nur die Ausführenden in ihrer selbst gewählten Auftrittskleidung, mit ihrer Mimik und ihren Gebärden. Damit 
Musik nicht bloß als fingerfertiges Kunststück erscheint oder die Konzentration auf die ästhetische Idee durch den sinnlichen Genuß abgelenkt wird, muß ein weiteres Überzeugungsmittel hinzukommen. Das Instrument soll ssingen`. Es soll durch eine menschenähnliche Lautäußerung beseelt werden. ${ }^{22}$ Darauf basiert auch das Konstrukt einer musikalischen spersonas. Um das zu erreichen, empfiehlt Bach den Musikern sowohl einen technischen Effekt: das Tastenvibrato, als auch ein mentales Training, nämlich »singend dencken « und "sich hernach selbst einen Gedanken vorsinge(n), um den rechten Vortrag desselben zu treffen" (Mattheson 1737).

Indessen ist das Grundproblem damit nicht behoben. Musik hat keine andere Stimme als die, die Künstler ihr geben, sei es im Gesang oder am Instrument. Der Körper der Virtuosen, ihre Anwesenheit und individuelle Physiognomie, ihre spezifische Gestik oder der nur zu diesem Körper gehörende einmalige Finger- und Bogendruck sind elementare Teile des realen Menschen und kein semiotisches Produkt. Die materiell eigenständigen und personell spezifischen Stimmen und Klänge lassen sich nicht im Sinne der Werkintention kodieren, und unvorhergesehene somatische Ausschläge wie Erröten oder Herzklopfen können zwar ignoriert, aber nicht abgestellt werden. Zudem bleiben bei der Flüchtigkeit des Objekts die Ausübenden sozusagen als sichtbare Zeugen der gerade erlebten klanglich-materiellen Bedeutung übrig und versichern uns, daß nicht nichts war. Zahlreiche Zeugnisse spiegeln den Zwiespalt musikalischer Erfahrung wider. Während Schiller sich (autonomieästhetisch korrekt) von Laura am Klavier sentkörpert und ins ästhetische Elysium erhoben wähnt, mag Goethe nicht trennen zwischen dem Zauber der Musik und der Verzauberung durch die Musikerin. „Da fühlte sich - o daß es ewig bliebe! - / Das Doppel-Glück der Töne wie der Liebe«, schreibt er der Pianistin und Komponistin Maria Szymanowska ins Stammbuch. ${ }^{23}$ Hier bleiben die Grenzen zwischen ästhetischem Erleben, Aufmerksamkeit und Abschweifen, eigener Erinnerung, Assoziation und Vorbildung, Phantasie und Projektion offen. Und sie lassen sich nicht schließen. Was sich hier im Gehirn der Rezipierenden abspielt, entzieht sich der Einflußnahme der Autoren.

Die musikalische Persönlichkeit, nach der eingangs gefragt wurde, entsteht demnach in der performativen Situation durch das Erarbeiten und Hervorbringen der Noten und die einstudierten instrumentalen Ausdrucksmöglichkeiten, in die eigenes emotionales Erleben, Erfahrung mit anderen Interpretationen und kulturelle Prägungen einfließen. Das Gelingen der Aufführung hängt indessen auch vom Einverständnis des Publikums ab, das seinerseits bestimmte, durch musikalische Konventionen und Hörgewohnheiten abgerichtete Erwartungen mitbringt, die gleichwohl auch zeitbedingten Moden unterworfen sind. Dafür gibt die gewandelte Aufführungspraxis und Rezeption von sAlter Musikı ein aktuelles Beispiel. Der Vibratoton, längst konventionell verschlis$\operatorname{sen}^{24}$, wurde durch präzise Tonhöhen ersetzt. Innerhalb weniger Jahrzehnte wechselte dabei die Praxis (und das Image) weg von spröder hermetischer Ferne hin zu einer warmen, körperhaften Empfindung. Der (weitgehend) vibratolose Ton wirkt aufgrund des Verzichts auf Stimmähnlichkeit zwar ätherischer als der konventionelle, er bietet nach Cumming aber mehr Direktheit und "gentle intimacy« (129).

Für das Kunsterleben der Hörer und der Ausführenden wie für den Aufführungsverlauf selber spielt die unmittelbare Wechselwirkung von Ausübenden und Hörern eine zentrale Rolle. Die Aktivität dieser im Konzertsaal spürbaren physischen und mentalen sfeedback-Schleife، (Fischer-Lichte) läßt sich in besonderen Fällen selbst hirnphysiologisch nachweisen. Konsensuell zur Hör- und Sehaktion können bei einschlägig vorgebildeten Rezipienten auch Bewegungsareale im Gehirn aktiv werden, in denen musikalisches Handlungswissen gespeichert ist. ${ }^{25}$ Hinzu kommt bei Ausübenden und Komponierenden noch ein spezifischer Rückkopplungseffekt zwischen der haptischen Aktivität am Instrument und dem klanglichen Widerhall, der seiner- 
seits das Spiel (und die Erfindung) beeinflußt, wie György Ligeti am Beispiel seiner Klavieretüden beschrieben hat. Zwar wird durch die heutige Alltagssituation, nämlich das Abspielen elektronisch gespeicherter Musikdaten, die körperliche Kunsterfahrung wesentlich modifiziert, da die sfeedback-Schleife` wegfällt, sie ist aber nicht grundsätzlich aufgehoben. Der spezifische Ton der Interpreten, die hörbaren Anteile ihrer Gestik, das Timbre und die Dynamik, auf die wir Hörer emotional reagieren, übertragen sich - mit gewissen $\mathrm{Ab}$ strichen - auch durch Lautsprecher. Im klassisch-romantischen Repertoire ergänzen wir ohnehin die aus vielfacher Erfahrung im Gedächtnis bereits bekannte Konzertsaalsituation, selbst dann, wenn die räumlichen Effekte der Einspielung keine Saalakustik abbilden, sondern durch digital hergestellte Illusionsräume entstehen.

Unter dem Begriff ‘Körpersprache` wird allgemein ein ganzes Bündel nonverbaler Verhaltensweisen zusammengefaßt, die bei der kommunikativen Interaktion und beim Gefühlsausdruck eine zentrale Rolle spielen: Mimik, Gesten und Gebärden ebenso wie der Klang des Gesprochenen. Hier lassen sich universale und kulturspezifische Codes unterscheiden. Die Nutzung universaler Codes gehört zum Alltagswissen, während die Auflösung kulturspezifischer Gesten und Gebärden eine besondere Erforschung ästhetischer Konventionen erfordert. Zur Beschäftigung mit älterer Musik gehört historisches Wissen über die zeitgenössischen kulturellen Diskurse, um Körperbewegungen, Blickverhalten, Mimik und Berührungsverhalten (Haptik) einschätzen und analysieren zu können. Ihre Interpretation kann nur über eine Auseinandersetzung mit der historischen Zeit und deren stilistischen Besonderheiten erfolgen. In die Aufführungen fließen indessen (auch unbeabsichtigt) intertextuelle Erfahrungen und Kenntnisse ähnlichen Stils mit ein. Im Verlauf der Aufführung können sowohl spontane als auch bewußt einstudierte Gesten und Gebärden beobachtet werden. ${ }^{26}$ Gesten und Gebärden sollen die gefühlte emotionale Qualität des
Sounds unterstützen. Musizierende agieren hier innerhalb eines begrenzten Spektrums auf der Basis bestimmter Wahlmöglichkeiten, die definiert sind durch die Vorgaben in der Partitur, Aufführungskonventionen und den Stand der eigenen Auseinandersetzung mit der Zeit und dem Repertoire. Beim Musizieren stekken allerdings die durch die instrumentaltechnischen Erfordernisse des Spiels gezogenen Grenzen den Spielraum gestischer Möglichkeiten ab. Verschriftlichte Gesten in der Partitur finden sich in Form ikonischer Zeichen, wie manche melodische Manieren, als instrumentalspezifische Figurationen (deren Eigenart sich bei der Übertragung auf andere Instrumente zeigt, wie in den diversen Variationen von Paganinis Violin-Capricci für Klavier) oder als Ausdrucksgesten. Im Moment der Aufführung werden sie durch spezifische körperliche Aktionen wie etwa Finger- und Bogendruck, Tempo, Schattierungen in expressives Handeln umgewandelt individualisiert. Probleme, musikalische Gesten und Gebärden in die Analysen einzubeziehen, entstehen dadurch, daß sie selten klar erkennbar in der Partitur auftauchen. Doch auch in der Aufführung lassen sie sich nicht eindeutig als vorgeschriebene oder spontan ausgeführte Zeichen kategorisieren, weil die Variantenbreite ihrer individuellen Ausführung unbegrenzt ist. Performative Gesten hat man bereits im 18 . Jahrhundert eingehend diskutiert, während die Frage nach den verschriftlichten Körperspuren verstärkt in literaturwissenschaftlichen Diskursen und noch recht zaghaft in neueren musikwissenschaftlichen Ansätzen der letzten Jahrzehnte untersucht wird. Hier kann die Semiotik einen Weg bieten, um alle Aspekte auf einer einheitlichen Ebene zu diskutieren.

\section{Zeichen}

In den letzten Jahren sind die semiotischen Schriften von Charles S. Peirce wieder mehr ins Blickfeld von Kulturanalysen gerückt. ${ }^{27}$ Diese neue Peirce-Renaissance hängt damit zusammen, daß der Einsatz von Zeichentheo- 
rie mit einem deutlich gewandelten Erkenntnisinteresse einhergeht: weg von der Konzentration auf das schriftlich fixierte Werk, hin zum erklingenden performativen Ereignis. Obwohl die räumliche Disposition von Orchester und Publikum bei Aufführungen des klassisch-romantischen Repertoires im Prinzip gleichgeblieben ist, geht es nicht mehr vorrangig um die ästhetische Distanz, die für das Erkennen von Formen notwendig ist ${ }^{28}$, sondern um die Ausbreitung von Sound und den subjektiven Anteil der Ausführenden wie der Zuhörer und -hörerinnen an der Konstituierung des Werks. In der Strukturalismus-Begeisterung der 70er und 80er Jahre bot die Zeichentheorie vor allem eine Chance, die musikalische Analyse vom klebrigen Ballast einer unkontrollierbaren Hermeneutik zu befreien und den Musikdiskurs stärker an die Kognitionswissenschaften anzubinden. Die Zeichentheorie ${ }^{29}$ verfügt über eine abstrahierende Metasprache aus logisch-algebraischen Symbolen, mit der wortsprachliche Bedeutungen, die unvermeidlich und stillschweigend durch den kulturellen Kontext bereits mitgeliefert werden, ersetzt werden können. Zudem soll damit ein Einfühlen in die Autorenposition verhindert werden. Analog zur linguistischen Sprachanalyse wird eine auf den musikalischen Text bezogene Semiotik zunächst vor allem dazu genutzt, sich auf die formalen Operationen zu konzentrieren. Daher gilt es, musikalisch relevante Einheiten im Stück zu klassifizieren und zu systematisieren, um Strukturen erkennen, paradigmatische und syntagmatische Verläufe bestimmen und komparative Verknüpfungen mit außermusikalischen Phänomenen beziehungsweise anderen Künsten vornehmen zu können. ${ }^{30}$ Den Fokus bildet die Suche nach einer allgemeinen, objektiven smusikalischen Grammatikı, deren Repräsentationen von gebildeten Hörern auch erkannt werden sollen. Auf dieser Vorstellung fußen auch noch die Generative Theory of Tonal Music von Lerdahl / Jackendoff und die differenzierteren narrativen Analysen von Eero Tarasti. Inzwischen hat sich das Interesse von diesem als einseitig empfundenen szientifischen Ansatz der Produktions- und Rezeptionsästhetik auf eine einheitliche Sinneserfahrung von Musik verlagert. Fragen nach der Rolle des Performativen, nach emotionalem Erleben, der Wirkung, Aneignung, Verarbeitung von Musik sowie der Funktion des Gedächtnisses dabei stehen inzwischen im Vordergrund. Parallel dazu läßt sich eine pragmatische Wende in der hermeneutischen Diskussion erkennen, wenn die Entstehung von Bedeutung mit der performativen Erfahrung verknüpft wird.

Heute, wo die Rückgewinnung von Körperlichkeit und Präsenz eine so zentrale Rolle in den ästhetischen Diskursen spielt, überzeugt Peirces pragmatischer Zugriff vor allem dadurch, daß stets ein Bezug zum Konkreten gewahrt wird. Seine Zeichentheorie scheint deshalb besonders gut für Musik geeignet zu sein, weil ihr Grundkonzept auf einem transitorischen Ansatz beruht. Peirce geht von einer fließenden, gleichsam vorbewußten Erfahrung als erstem Eindruck aus, die der Erfahrung mit der immateriellen Flüchtigkeit von $\mathrm{Mu}$ sik ähnlich ist. Die Verflüchtigung des erklingenden Augenblicks bereitet jeder musikalischen Theorie Probleme. ${ }^{31}$ Nach Lidov vertritt Cumming sogar die These, Peirces Theorie sei von Musik geprägt. ${ }^{32}$ In dem Moment, wo man sich dieses ersten unmittelbaren Eindrucks bewußt wird, nämlich in einem zweiten Augenblick, ist der erste bereits Geschichte. Das Bewußtsein eines Eindrucks oder einer diffusen Empfindung wird indessen erst dann zu einer Erkenntnis, wenn in einem weiteren Schritt darüber nachgedacht wird, in welcher Beziehung der erste Gedanke zu dem unmittelbaren sinnlichen Eindruck steht. Ein gehörter Klang läßt nichts zurück als eine leere Stelle. In dem Moment, wo man ihn bewußt registriert, ist er auch schon verklungen. Aus der Erinnerung wird dann versucht, das sinnlich Erfahrene zu formulieren. Umberto Eco liefert für den prozessualen Erkenntnischarakter von Peirces Theorie ein anschauliches Beispiel. Ein undeutliches Etwas im Dunkeln wird registriert. Man reagiert mit erhöhter Aufmerksamkeit, schaut genauer und wendet darauf die aus früherer Erfahrung resultierende 
kulturelle Einheit `Katze` an. Der primäre Eindruck kann dann sprachlich interpretiert werden: »Ich habe eine Katze gesehen. ${ }^{33}$ Diese drei Kategorien (Peirce) des Erkenntnisprozesses, nämlich die sinnliche Erfahrung, ihre analytische Abstraktion und die formale Bestimmung, korrelieren mit den drei Stufen des Prozesses der Zeichenbildung: dem Zeichen an sich, seiner Beziehung zum Objekt und der Interpretation dieser Beziehung. Peirces $১ \mathrm{Ob}-$ jekt garantiert den Bezug zur Wirklichkeit.

Peirces Zeichen verfügen über den Vorteil, daß sie intermedial offen sind und daher komparatistische Ansätze unterstützen. Sie gelten für Sprache ebenso wie für »Gesten, Spuren im Schnee oder neurophysiologische und physikalische Signale $\ll^{34}$ und eben auch für Klänge. Klänge sind konkret erfahrbare akustische Ereignisse, die einmal für sich stehen und darüber hinaus im Kontext einer Rede oder Musik Bedeutung gewinnen können. Dieser Aspekt ist in der triadischen Zeichenstruktur von Peirce angelegt. Dabei fällt die Beziehung zwischen Zeichen und Objekt unterschiedlich aus. Wenn zwischen Zeichen und Objekt eine qualitative Entsprechung besteht (Ähnlichkeit, Analogie), so gilt es in Peirces Theorie als Ikon. ${ }^{35}$ Dazu gehören sowohl onomatopoetische Nachahmungen (musikalische Gewitter oder Vogelzwitschern) als auch viele Madrigalismen. Besteht eine aktuale oder sals-ob، repräsentierte Beziehung, so ist das Zeichen ein Index (wie bei der musikalischen 'Seufzer`-Figur), während die Objekt-Beziehung beim Symbol erst durch Konvention zustande kommt, wie in Notationssystemen. Die triadische Struktur von Peirces Zeichendefinition scheint auch deswegen ideal für artifizielle Musik zu sein, weil sie einmal von einer Identität als Resultat von Verknüpfung ausgeht und zweitens einen zeitlich unumkehrbaren Ablauf von »mindestens zwei Erfahrungsereignissen« vorsieht. Das erste betrifft die Relation zwischen der symbolischen Notenschrift der Partitur und der erklingenden Musik. Der zweite Aspekt betrifft den Entwurf eines musikalischen Werkes, dessen klingende Realisierung in die Zu- kunft projektiert ist. Musik wird erst im Erklingen realisiert. Jede weitere Aufführung bedeutet eine neue Überführung der Symbolschrift in unsere Wirklichkeit, die die vorige mit einbezieht. In der Offenheit von Peirces Zeichenkonzept kann der erste triadische Schritt wiederum zum Ausgangspunkt eines neuen sinnlichen Erfahrens, also eines neues Zeichens werden und so einen Prozeß ständiger Weiterproduktion auslösen. Metaphorisch läßt sich dieser Vorgang auf die Aufführungstradition von artifizieller Musik übertragen.

Im Fall von Klang muß allerdings aufgrund der materiellen Eigenschaften von erklingender Musik (Schallwellen) mit einem symbolischen Objekt operiert werden. Cumming setzt hier ein »object of perception« (70) als semiotisches Objekt ein, das an das Medium des produzierten Tons gebunden ist, und differenziert damit die Anwendung der Zeichen noch einmal. Die Analogien zwischen einem musikalischen Zeichen und dem gehörten Objekt entstehen ja nicht direkt, sondern sie beziehen sich auf den Interpretanten. So kann eine Violine ssingen', sofern sie bestimmte Qualitäten der menschlichen Stimme (Vibrato, Modulation) imitiert oder exemplifiziert. Gleichwohl wird dieses 'Singen in der Regel nur erkennen, wer zu einer Gruppe von `Klassikı-Rezipienten gehört, die sich bereits einig darüber ist, daß Violinen überhaupt singen können. Diese Gruppe verständigt sich schon in einer bestimmten Metaphorik (daß Instrumente zu singen vermögen, stellt niemand in Frage) und pflegt damit einen spezifischen Diskurs, der durch gemeinsame Musikpräferenzen, aber auch durch die hartnäckige Konstanz bestimmter Auffassungen oder Bilder festgeschrieben ist. Zur Werteskala des Urteils über das Gelingen von Instrumentalmusikaufführungen gehört seit dem 18. Jahrhundert die Vorstellung eines instrumentalen Singens. Im Unterschied zum Realitätsgrad von Peirces Objekten läßt sich das Fehlen oder Vorhandensein gesanglicher Qualitäten eines Violinspiels allerdings kaum meßbar nachweisen. Es entspricht eher einer Suggestion als einer objektivierbaren sinnlichen Erfahrung. Mit 
dem Üben wird diese Präferenz des snatürlichen Singens` als kulturell determiniertes Zeichen im Handlungswissen gespeichert. Das alles gehört zu einem Erfahrungsschatz, der in Form von Intuition wieder an die Oberfläche tritt. Dieser Pool ist teilweise individuell und teilweise kollektiv, weil darin Erfahrungen gesammelt sind, die man nicht allein mit Musik macht. Dazu kommt die bewußte oder unbewußte Auseinandersetzung mit Vorbildern, die zeichentheoretisch wiederum neue sinnliche Erfahrungen und damit Ausgangspunkt für einen weiteren triadischen Prozeß sind. »Wenn Sie so singen wie Ihr Vorbild, singen Sie nicht mit Ihrer eigenen Muskulatur. ॥ $^{36}$ Dieser paradoxe Satz von Thomas Quasthoff zeigt anschaulich, wie komplex die Interpretationsvorgänge gestaltet sind. Auf dieser Basis läßt sich schließlich ein neuer Ansatz für eine Interpretationstheorie gewinnen.

Peirces Zeichentheorie ist nicht auf Kunst, sondern auf die Erkenntnis von Wirklichkeit gerichtet. Im Gegensatz zu Chomsky, der Sprachstrukturen zu überschaubaren Diagrammen abstrahiert, entfaltet Peirce ein sehr differenziertes und nicht immer leicht verständliches Zeichen- und Bezugssystem. Die musikwissenschaftliche Erwartung an die Pragmatik seiner Theorie ist meist zu hoch, die Frustrationsschwelle dagegen zu niedrig angesetzt. Wenn man tatsächlich von Peirces Theorie profitieren möchte, so ist das ohne Anstrengung nicht zu haben. Die Auseinandersetzung damit schärft aber die Sensibilität nicht nur für die performativen Qualitäten, sondern für das komplexe System aus Notenzeichen und akustischem Ereignis, Selbstkonstitution und Erfahrung, kulturellen Codierungen und persönlichen Entscheidungen, die den kommunikativen Austausch durch, mit und über Musik bereichern.

\section{Nachruf}

Vieles, was bei Cumming in unglaublicher Fülle bloß angerissen und als frische Idee projektiert ist, würde man gern an die Autorin zurückverweisen, damit sie ihre Entdeckungen weiter erforscht, sortiert, zusammenfaßt und verschriftlicht, so daß man die Ergebnisse bequem rezipieren kann. Doch Naomi Cumming ist am 6. Januar 1999 in Brisbane, Australien, gestorben. ${ }^{37}$ The Sonic Self wurde posthum im Jahr 2000 von der »Naomi Helen Cumming Foundation« herausgegeben. Die Autorin studierte Violine und Musiktheorie an den Universitäten von Tasmania und Melbourne, wo sie auch promovierte. Verschiedene Stipendien und Forschungsförderungen nutzte sie für umfangreiche Studien zu den Schwerpunkten Musiktheorie und -ästhetik, Musikpsychologie sowie Semiotik und Musikpädagogik. Ihre letzte Stelle als Senior Lecturer in Music an der Universität von Queensland konnte sie nicht mehr antreten. Aus der beeindruckenden Liste von Publikationen möchte ich den Beitrag "The Subjectivities of ,Erbarme dich « (Music Analysis 16, 1997, S. 5-44) herausheben, der als bester Artikel des Jahres von der Internationalen Gesellschaft für Musiktheorie ausgezeichnet wurde. Bevor ich nun in Versuchung gerate, anhand ihrer im Buch und im Internet veröffentlichten Portraits mich in Mutmaßungen über eine fragile junge Frau zu versteigen, stoppt mich die Autorin selber mit ihren überaus klugen und kritischen Bemerkungen zur Gender-Analyse von Rezensionen männlicher und weiblicher Performer. Zwei wesentliche Aspekte pickt sie heraus, nämlich einmal die Projektion des musikalischen Charakters auf die Performer (und umgekehrt!), und zweitens die Beobachtung, daß von weiblichen Performern selbstverständlich emotionale Qualitäten erwartet werden. ${ }^{38}$ Vor allem zeigt ihr Buch ein fundiertes, spartenübergreifendes Wissen sowie die Fähigkeit, ungewöhnliche Verknüpfungen vorzunehmen und damit neue Perspektiven zu entwerfen. Alles, was an den Themen ihres Buches so brennend interessiert, bleibt nun uns selber als Aufgabe überlassen. Also: an die Arbeit!

Janina Klassen 


\section{Anmerkungen}

1 An dieser Stelle möchte ich Anne-Kathrin Rist und Volker Jäger für intensive Diskussionen und beharrliches Nachfragen ebenso herzlich danken wie Oliver Schwab-Felisch.

2 Neuere sprachtheoretische Ansätze finden sich bei Krämer / König 2002; Fischer-Lichte 2004.

3 Lakoff / Johnson ${ }^{3} 2003$, 44f.

4 la Motte 1982, 182ff., besonders 232; Vgl. hierzu Brandstätter 1990, 110ff.

5 Auf diese Hemmschwellen eingehend baut Christiane Tewinkel ihre Essays zum Klassik-Hörer-Verhalten, die am Ende dann doch einer subtilen Erziehung zur strukturellen Hörer-Haltung recht nahe kommen (Tewinkel 2004).

6 Doku-Fernseh-Serien wie »Schwarzwaldhaus 1902« oder »Windstärke 8 - Das Auswandererschiff 1855 « demonstrieren anschaulich das Mißlingen des Versuchs, sich in die historische Zeit einzufühlen.

7 Vgl. die Zusammenfassung des Problems bei Sanio 1998, besonders 484-487.

8 Cumming weist die generalisierenden Regelsysteme allgemein der Musiktheorie, die Diskussion über den Sinn (meaning) von Musik der Ästhetik zu und beruft sich dabei auf Riemann (168f.). Diese Trennung dürfte in so zugespitzter Form nicht bestehen. Ich benutze hier 'Sinn in bezug auf selbstreferentielle Strukturen von Musik.

9 Lerdahl / Jackendoff 1983; Raffman 1993; Meyer 1956; Narmour 1990.

10 Vgl. die unterschiedlichen Sichtweisen von Apel 1967 und Pape 2004.

11 Trabant 1986, 206.

12 Das war der Ausgangspunkt der von Sybille Krämer initiierten Ringvorlesung "Gibt es eine Sprache hinter dem Sprechen?».

13 In der von Frank Gertich konzipierten soirée electronique in Berlin, wo Amachers Stück am 2. September 2004 zu hören war, wurde per Ansage vorher versichert, es ginge nichts kaputt.

14 So der Titel von Goebel 1998.

15 Dahlhaus 1977, 13

16 Fischer-Lichte 2004, 171ff. Die von Cumming angestrebte Aufhebung der body-mind-Opposition ist in diesem Ausdruck zusammengefaßt.
17 Bach 1753/62, 122.

18 Fischer-Lichte, 2004, 138.

19 Ebd., 133. - Siehe auch C[arola] S[urkamp], Art. "Possible-worlds theory (PWT), in: Metzler-Lexikon Literatur- und Kulturtheorie, hg. von Ansgar Nünning, Stuttgart und Weimar ${ }^{2} 2001,517 f$.

20 Richard Schechner bespricht am Beispiel von Laurence Olivier als Othello die notwendige »monitoring capacity des Schauspielers (35).

21 Bach 1753/62, 122.

22 Das aus der persuasiven Rhetorik stammende Konzept beseelter Rede überträgt Mattheson auf die »Klang-Rede«. Vgl. Mattheson 1737, $60 \mathrm{ff}$.

23 Friedrich Schiller, "Laura am Klavier«, zit. nach Lütteken 1998, 543f; Johann Wolfgang Goethe, »Aussöhnung», in: Ders., Werke Bd. 1, Frankfurt am Main o.J., 209.

24 »Warum hört sich Kunstgesang so eigenartig an? fragt Christiane Tewinkel am Beispiel der Oper. (Tewinkel 2004, 148).

25 Auf dieser Basis funktioniert mimetisches Lernen. Vgl. zur sfeedback-Schleife، Fischer-Lichte 2004, 59. Den neurophysiologischen Vorgang hat Eckart Altenmüller anschaulich dargestellt: Eckart Altenmüller, 2005, 331f.; zur Funktion von Spiegelneuronen vgl. Roth 2003, 450.

26 Einen Sonderfall bildet die aus der Theorie des Epischen Theaters stammende ,Gestikı-Definition von Bertolt Brecht, auf die nicht näher eingegangen wird.

27 Charles S. Peirce hat keine geschlossene Theorie hinterlassen, sondern etwa 800 Publikationen in 24 Fächern; dazu kommen 12.000 Lexikonartikel. In seinem Nachlaß fanden sich noch einmal ca. 100.000 Seiten. Seine Zeichentheorie ist keine spezifisch linguistische, sondern eine philosophisch fundierte, die alle Bereiche des Lebens betrifft. Daher existieren eine Vielzahl von Zeichendefinitionen aus unterschiedlichen erkenntnistheoretischen Perspektiven. Auf deutsch ist eine Auswahl von Schriften zwischen 1865 und 1914 erschienen (Peirce 2000). Es empfiehlt sich, auch die Einführungen von Helmut Pape (2004) und Winfried Nöth (2000) hinzuzuziehen.

28 Vgl. Dahlhaus 1978, 281. 
29 Vgl. Naomi Cumming, Art. "Semiotics (Semiology)«, in: The New Grove Dictionary, Ausg. 2001. Der entsprechende Art. »Zeichen« von Christian Kaden, in: Die Musik in Geschichte und Gegenwart, zweite Ausgabe, Sachteil, geht von einem umfassenden, allgemeinen Zeichenbegriff aus und bietet eine kulturhistorische Perspektive.

30 Daß dieses Ergebnis oft auch mit traditioneller motivisch-thematischer Analyse erreicht werden kann, vermerkt Nicholas Cook nüchtern am Beispiel von Jean-Jacques Nattiez. Zit. nach Cook 1994, $151 f$.

31 Dader Augenblick unwiderruflich verloren ist, hat, nach Johann Beer, der Komponist sozusagen nur eine Chance, und die mußgelingen. Beer 1719, 42.
32 Cumming 2000, XVII.

33 Umberto Eco ${ }^{9} 2002,84$.

34 Pape 2004, 117.

35 Zu Recht unterstreicht Mieke Bal, daß mit »icon« nicht ausschließlich Bilder gemeint seien. Bal 2002, $20 \mathrm{ff}$.

36 Thomas Quasthoff, „Ich sage schon immer, was ich denke«, in: Berliner Philharmoniker. Das Magazin, hg. von der Berliner Philharmonie $\mathrm{GmbH}$ (Mai, Juni 2004), 13.

37 Siehe http://www.nhcf.org.au/naomibiography. html vom 17. August 2004.

38 Cumming 2000, 23f.; am Beispiel aus Rezensionen der Fachzeitschriften Strads und Strings.

\section{Literatur}

Altenmüller, Eckart, (2005), „Was hört das Auge, was sieht das Ohr?", in: Hören und Sehen - Musik audiovisuell, Mainz: Schott, 326-333.

Apel, Karl-Otto (1967), Der Denkweg von Charles S. Peirce. Eine Einführung in den amerikanischen Pragmatismus, Frankurt am Main: Suhrkamp.

Bach, Carl Philipp Emanuel (1753/62), Versuch über die wahre Art das Clavier zu spielen, Berlin, Neudruck Leipzig und Wiesbaden: Breitkopf \& Härtel ${ }^{6} 1986$.

Bal, Mieke (2002), Kulturanalyse, Frankfurt/M.: Suhrkamp 2002.

Barthes, Roland (31991), »Die Rauhheit der Stimme», in: Aisthesis. Wahrnehmung heute oder Perspektiven einer anderen Ästhetik, hg. von Karlheinz Barck, Peter Gente, Heidi Paris und Stefan Richter, Leipzig: Reclam, 299-309.

Beer, Johann (1719), Musicalische Discurse durch die Prinzipia der Philosophie deducirt, Nürnberg.

Brandstätter, Ursula (1990), Musik im Spiegel der Sprache. Theorie und Analyse des Sprechens über Musik, Stuttgart: Metzler.
Cook, Nicholas (1987), A Guide to Musical Analysis, London: Dent.

Dahlhaus, Carl (1977), Grundlagen der Musikgeschichte, Köln: Gerig.

— (1987), „Über den Zerfall des Werkbegriffs«, in: Ders., Schönberg und andere, Mainz: Schott, 279-290.

Eco, Umberto ( $\left.{ }^{9} 2002\right)$, Einführung in die Semiotik, München: Fink.

Fischer-Lichte, Erika (2004), Ästhetik des Performativen, Frankfurt am Main: Suhrkamp.

Krämer, Sybille / Ekkehard König (Hg.) (2002), Gibt es eine Sprache hinter dem Sprechen? Frankfurt am Main: Suhrkamp.

Lakoff, George / Marc Johnson (1980), Metaphors We Live By, Chicago: University of Chicago Press, dt. als: Leben in Metaphern. Konstruktion und Gebrauch von Sprachbildern, Heidelberg: Carl-Auer Systeme Verlag ${ }^{4} 2004$.

La Motte-Haber, Helga de (1982), „Musikalische Hermeneutik und empirische Forschung", in: Systematische Musikwissenschaft, hg. von Carl Dahlhaus und Helga de la Motte-Haber, Laaber: Laaber, 171-244. 
Lerdahl, Fred / Ray Jackendoff (1983), A Generative Theory of Tonal Music, Cambridge, Mass.: MIT Press.

Lütteken, Laurenz (1998), Das Monologische als Denkform in der Musik zwischen 1760 und 1785, Tübingen: Niemeyer.

Mattheson, Johann (1737), Kern melodischer Wissenschafft, Hamburg, Reprint Hildesheim: Olms 1976.

Meyer, Leonard B. (1956), Emotion and Meaning in Music, Chicago: University of Chicago Press.

Narmour, Eugene (1990), The Analysis and Cognition of Basic Melodic Structures. The Implication-Realization Model, Chicago: University of Chicago Press.

Nöth, Winfried (2000), Handbuch der Semiotik, Stuttgart und Weimar: Metzler.

Pape, Helmut (2004), Charles S. Peirce zur Einführung, Hamburg: Junius.

Raffman, Diana (1993), Language, Music and Mind, Cambridge, Mass.: MIT Press.
Peirce, Charles S. (2000), Semiotische Schriften, hg. und übers. von Christian J.W. Kloesel und Helmut Pape, 3 Bde., Frankfurt a.M.: Suhrkamp.

Roth, Gerhard (2003), Fühlen, Denken, Handeln. Wie das Gehirn unser Verhalten steuert, Frankfurt a.M.: Suhrkamp.

Sanio, Sabine (1998), "Jenseits der Geschichte? Das Verhältnis der Geschichtswissenschaften zur Gegenwartskunst", in: Musikwissenschaft zwischen Kunst, Ästhetik und Experiment, Festschrift Helga de la MotteHaber zum 60. Geburtstag, hg. von Reinhard Kopiez u.a., Würzburg: Königshausen \& Neumann, 483-94.

Tewinkel, Christiane (2004), Bin ich normal, wenn ich mich im Konzert langweile? Eine musikalische Betriebsanleitung, Köln: DuMont

Trabant, Jürgen (1986), Apeliotes oder Der Sinn der Sprache, München: Fink. 